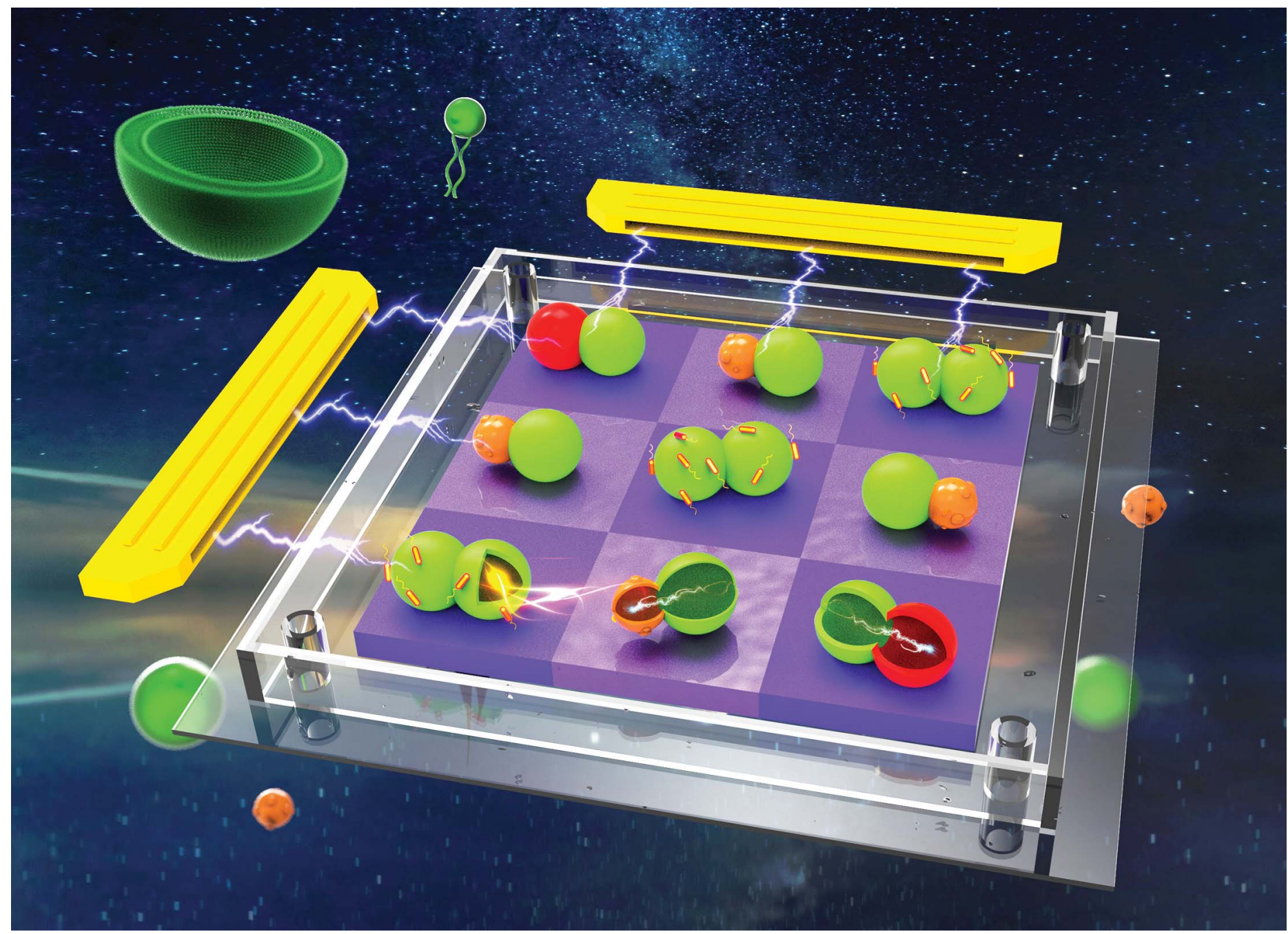

Showcasing research from Professor Xiaojun Han's laboratory, School of Chemistry and Chemical Engineering, Harbin Institute of Technology, Harbin, China.

Chemical communication in spatially organized protocell colonies and protocell/living cell micro-arrays

Micro-arrays of discrete or hemifused giant unilamellar lipid vesicles (GUVs) with controllable spatial geometries, lattice dimensions, trapped occupancies and compositions are prepared by acoustic standing wave patterning, and employed as platforms to implement chemical signaling in GUV colonies and protocell/ living cell consortia. The methodology is straightforward, dynamic, adaptable in terms of micro-engineering, and readily amenable to in situ chemical manipulations possibly including non-equilibrium spatiotemporal sensing. It provides new opportunities in protocell research and bottom-up synthetic biology including the assembly of synthetic prototissues etc.

\section{As featured in:}

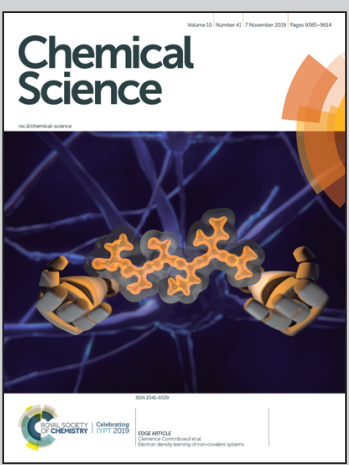

See Xiaojun Han,

Stephen Mann et al.,

Chem. Sci., 2019, 10, 9446. 
Check for updates

Cite this: Chem. Sci., 2019, 10, 9446

๑ All publication charges for this article have been paid for by the Royal Society of Chemistry

Received 9th September 2019

Accepted 11th September 2019

DOI: $10.1039 / \mathrm{c} 9 \mathrm{sc} 04522 \mathrm{~h}$

rsc.li/chemical-science

\title{
Chemical communication in spatially organized protocell colonies and protocell/living cell micro- arrays $\uparrow$
}

\author{
Xuejing Wang,,$^{\mathrm{ab}}$ Liangfei Tian, (D) $\dot{t}^{\mathrm{b}}$ Hang Du, ${ }^{\mathrm{a}}$ Mei Li, (D) ${ }^{\mathrm{b}}$ Wei Mu, ${ }^{a}$ \\ Bruce W. Drinkwater, (ID C Xiaojun Han (D) ${ }^{* a}$ and Stephen Mann (iD *b
}

\begin{abstract}
Micro-arrays of discrete or hemifused giant unilamellar lipid vesicles (GUVs) with controllable spatial geometries, lattice dimensions, trapped occupancies and compositions are prepared by acoustic standing wave patterning, and employed as platforms to implement chemical signaling in GUV colonies and protocell/living cell consortia. The methodology offers an alternative approach to GUV micro-array fabrication and provides new opportunities in protocell research and bottom-up synthetic biology.
\end{abstract}

\section{Introduction}

Giant unilamellar lipid vesicles $(>1 \mu \mathrm{m}, \mathrm{GUVs})^{1}$ have been used widely as a model cell system in biophysical research to investigate membrane mechanical properties, ${ }^{2}$ shape transformations, ${ }^{3}$ phase separation, ${ }^{4}$ budding and division, ${ }^{5,6}$ fusion, ${ }^{7-9}$ permeability, ${ }^{10}$ and protein/lipid bilayer interactions. ${ }^{11}$ GUVs have also been exploited as enzyme-based microreactors ${ }^{\mathbf{1 2}}$ and for the design and construction of lipidbased protocell models exhibiting diverse functions., ${ }^{\mathbf{5}, 12-18}$ Significantly, microarrays of single GUVs of uniform size for potential use in high-throughput biochemical and chemical analysis have been developed using microcontact printing ${ }^{\mathbf{1 9 2 0}}$ or microfluidics. ${ }^{\mathbf{2 1 , 2 2}}$ Herein, we demonstrate a novel approach to the direct fabrication of GUV micro-arrays based on the spontaneous trapping of lipid vesicles in an acoustic (ultrasonic) standing wave pressure field. Although multi-dimensional trapping of micro-particles, ${ }^{23,24}$ liquid droplets, ${ }^{25}$ cells ${ }^{23,24,26}$ and organisms ${ }^{23,24}$ in water have been achieved using acoustic radiation forces, GUV micro-arrays have not previously been acoustically trapped due to the lack of acoustic contrast between the lipid vesicles and the surrounding aqueous continuous phase. We circumvent this problem by using a sucrose/glucose density gradient across the vesicle membrane and engineer the acoustic standing wave to generate one dimensional (1D) or two

${ }^{a}$ State Key Laboratory of Urban Water Resource and Environment, School of Chemistry and Chemical Engineering, Harbin Institute of Technology, Harbin, 150001, China. E-mail: hanxiaojun@hit.edu.cn

${ }^{b}$ Centre for Protolife Research and Centre for Organized Matter Chemistry, School of Chemistry University of Bristol, Bristol, BS8 1TS UK. E-mail: s.mann@bristol.ac.uk

${ }^{c}$ Faculty of Engineering, University of Bristol, Bristol, BS8 1TR UK

$\dagger$ Electronic supplementary information (ESI) available. See DOI: 10.1039/c9sc04522h

\$ X. J. Wang and L. Tian contributed equally to this work. dimensional (2D) reconfigurable micro-arrays of GUVs with controllable spatial geometries (linear, square, rectangular, triangular) and lattice spacings. We demonstrate that microarrays consisting of single or multiple GUVs trapped at each zero-pressure point (node) of the acoustic pressure field can be fabricated by controlling the number of GUVs added to the acoustic trapping device and exploit the co-location of multiple GUVs to produce spatially arranged colonies ${ }^{27}$ of hemifused GUVs.

We use the micro-arrays of acoustically trapped GUVs for the spatial positioning and signaling of enzyme reactions. By trapping colonies of heterogeneous GUV populations containing either glucose oxidase (GOx) or horseradish peroxidase (HRP) we produce spatially distributed communities of synthetic protocells capable of localized enzyme-mediated chemical signaling triggered by a pore-forming peptide (melittin) inserted into the lipid membrane. We employ a similar strategy for the localization and killing of cancer cells ${ }^{28}$ by co-trapping clusters of GOx-containing GUVs and HepG2 cells. Finally, by co-trapping clusters of isopropyl $\beta$-D-thiogalactoside (IPTG)containing GUVs and bacterial cells (Escherichia coli) at the acoustic pressure nodes in the presence of melittin, we demonstrate a protocell/living cell transmitter/receiver arrangement for bacterial gene expression.

\section{Results and discussion}

Spherically shaped GUVs typically $25 \pm 14 \mu \mathrm{m}$ in diameter were prepared from 1,2-dioleoyl-sn-glycero-3-phosphocholine (DOPC) using an electroformation method (Fig. S1†). ${ }^{29}$ We used sucrose-containing GUVs dispersed in an isotonic glucose solution $(>25 \mathrm{mM})$ to generate a persistent density gradient $(>0.5 \%)$ with sufficient acoustic contrast for efficient and reproducible trapping in an ultrasonic standing wave pressure field (Fig. 1a, S2 and S3†). The protocells were trapped typically 
within 15 min to produce $1 \mathrm{D}$ rows, 2D square grids or triangular micro-arrays by engineering the acoustic pressure fields accordingly (Fig. $1 \mathrm{~b}-\mathrm{h}$ and $\mathrm{S} 4 \dagger$ ). In each case, the DOPC vesicles remained structurally intact and non-fused in the acoustic pressure field, and the centre-to-centre line spacings could be readily modulated by changing the operational frequencies (Fig. 1f, g and S5, S6 $\dagger$ ). Moreover, reversible dynamic reconfiguration of the GUV arrays from vertical to horizontal alignments or from 1D to 2D arrangements could be achieved within ca. $4 \mathrm{~min}$ by on/off switching of the corresponding pairs of piezoelectric transducers (Fig. S7 and Movie S1†). In general, switching off the acoustic field resulted in re-dispersal of the GUVs into the bulk solution typically within 5-10 min (Fig. S8†).

The level of protocell occupancy at each acoustic pressure node was adjusted by changing the GUV/node number ratio $\left(R_{\mathrm{G} / \mathrm{N}}\right)$ (Fig. $2 \mathrm{a}-\mathrm{c}$ and $\left.\mathrm{S} 9 \dagger\right)$. When $R_{\mathrm{G} / \mathrm{N}}$ was below 1.2, ca. 33\% of the nodes were occupied by single vesicles with the remaining sites being unoccupied (Fig. 2a and S9a †े). The occurrence of two vesicles per node reached a maximum value ( $c a .25 \%)$ when the $R_{\mathrm{G} / \mathrm{N}}$ ratio was $c a .2 .2$ (Fig. $2 \mathrm{~b}$ and $\mathrm{S} 9 \mathrm{~b} \dagger$ ), and multiple numbers of vesicles per node (ca. 76\%) were observed at $R_{\mathrm{G} / \mathrm{N}}$ values above 3.4 (Fig. 2c and S9c†). Clusters of multiple GUVs were predominantly trapped at $R_{\mathrm{G} / \mathrm{N}}>3.4$ (Fig. $2 \mathrm{~d}$ ), enabling the co-location of mixed protocell populations at each acoustic pressure node (Fig. S10†). We used this approach as a step towards the periodic arrangement of spatially separated colonies of protocells capable of structural integration and functional activation. For this, we exploited local contact points between the GUVs as foci for the $\mathrm{Ca}^{2+}$-induced hemifusion of the co-localized protocells in the presence of the acoustic field (Fig. S11†). Hemifusion of the vesicles resulted in mixing of the membrane lipids (Fig. S12†), while no lipid exchange was
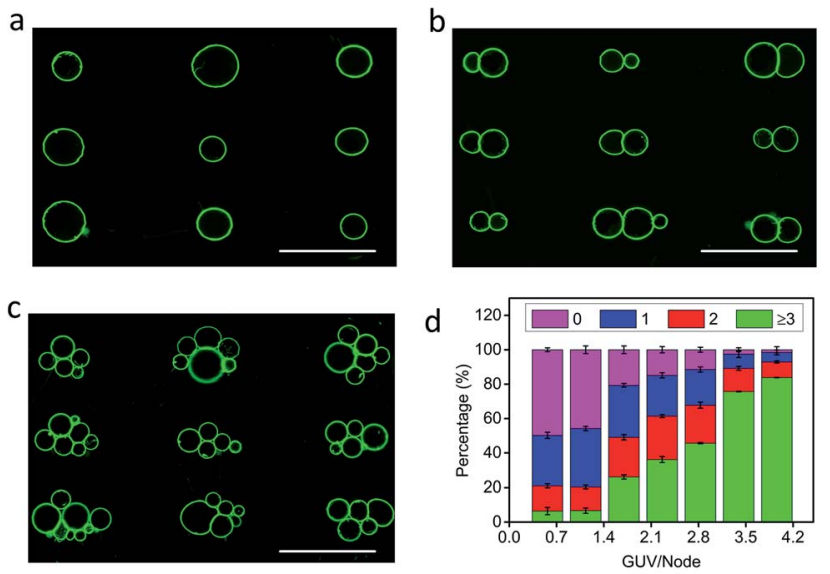

Fig. $2(\mathrm{a}-\mathrm{c})$ Representative fluorescence microscopy images of acoustically trapped rectangular micro-arrays consisting of single (a), double (b) and multiple (c) GUVs at each pressure node; samples prepared using GUV/node number ratios $\left(R_{\mathrm{G} / \mathrm{N}}\right)$ of $1.2(\mathrm{a}), 2.2$ (b) and 3.4 (c). All scale bars are $100 \mu \mathrm{m}$. (d) Plots of the percentage of unoccupied nodes (no GUVs, black) and occupied nodes containing single (red), double (blue) or multiple GUVs ( $\geq 3$, cyan) against $R_{\mathrm{G} / \mathrm{N}}$. In total, three independent replicates were conducted at each GUV/node number ratio $\left(R_{\mathrm{G} / \mathrm{N}}\right)$. In each replicate, 500 nodes were counted. Error bars represent the standard deviation.

observed in the absence of $\mathrm{Ca}^{2+}$ ions (Fig. S13†). Moreover, in comparison to the non-fused GUVs, the hemifused GUV clusters remained spatially fixed at the lattice positions when the acoustic pressure field was switched off. This allowed us to prepare arrays using a two-step patterning procedure that contained co-located clusters of non-fused and hemifused GUVs that exhibited protocell-selective dynamical responses to changes in the acoustic pressure field (Fig. S14 and Movie S2 $\dagger$ ).
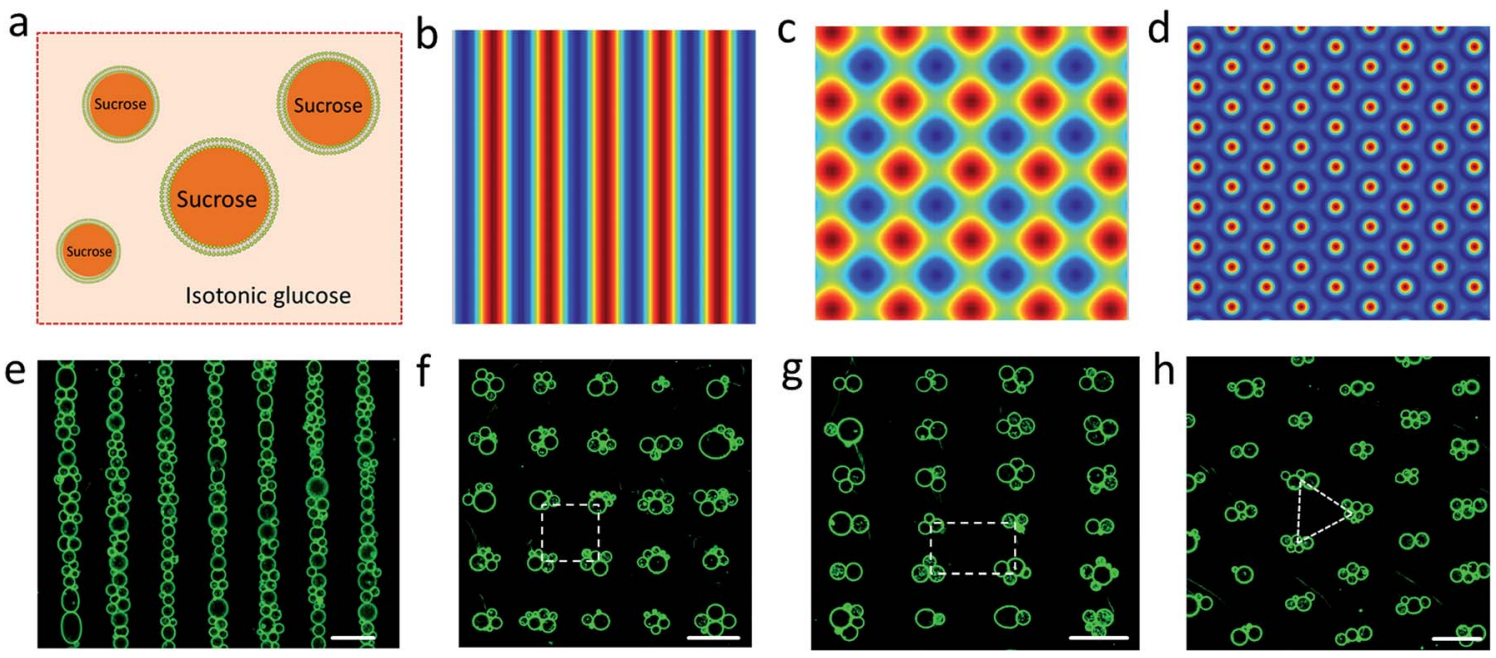

Fig. 1 (a) Schematic of sucrose-containing GUVs in isotonic glucose solution used for acoustic trapping. (b-d) Simulation of the acoustic pressure distribution in the acoustic trapping device for one pair of piezoelectric transducers (PZTs) (b), two pairs of PZTs with a square arrangement (c), and three pairs of PZTs with hexagonal arrangement (d); high pressure (blue, antinodes), low pressure (red, nodes). (e-h) Representative fluorescence microscopy images of micro-arrays of green GUVs (5\% NBD-PE) produced in a 1D acoustic pressure field (6.71 MHz, $10 \mathrm{~V})(\mathrm{e}) ; 2 \mathrm{D}$ field $(6.69 / 6.71 \mathrm{MHz}, 10 \mathrm{~V})(\mathrm{f}) ; 2 \mathrm{D}$ field $(5.06 / 9.13 \mathrm{MHz}, 10 \mathrm{~V})(\mathrm{g})$, and 2D field (6.70/6.71/6.72 MHz, $10 \mathrm{~V})(\mathrm{h})$. Centre-to-centre line spacings: ca. $110 \mu \mathrm{m}$ (e), $110 \times 110 \mu \mathrm{m}$ (inter-vesicle distance, ca. $50 \mu \mathrm{m}$ ) (f), $146 \times 81 \mu \mathrm{m}(\mathrm{g})$. All scale bars are $100 \mu \mathrm{m}$. 


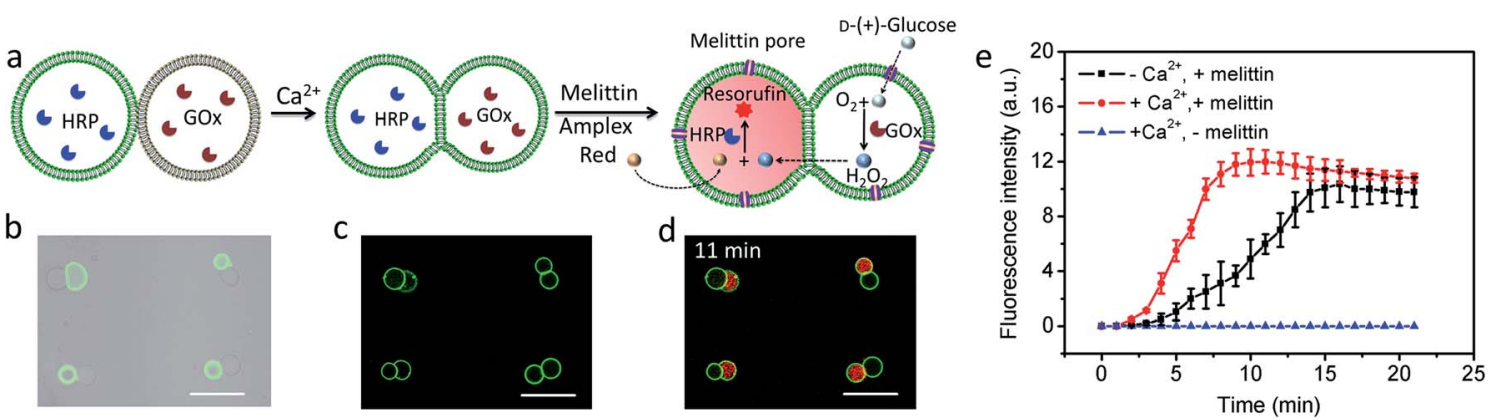

Fig. 3 (a) Schematic of a hemifused heterogeneous pair of acoustically trapped GUVs containing either HRP or GOx, followed by addition of melittin, Amplex red and glucose. (b) Superimposition of bright field and fluorescence microscopy images of a rectangular micro-array consisting of colonies of co-trapped NBD-PE-labeled HRP-containing GUVs (green fluorescence) and unlabeled GOx-containing GUVs. (c) As for (b), but after $\mathrm{Ca}^{2+}$-induced hemifusion. (d) As for (c), but superimposition of red and green fluorescence image recorded $11 \mathrm{~min}$ after addition of melittin and Amplex red. All scale bars are $50 \mu \mathrm{m}$. (e) Time-dependent changes in mean fluorescence intensity for GOx/HRP-mediated formation of resorufin in acoustically trapped micro-arrays comprising melittin-functionalized hemifused multiple GUVs (red), melittin-functionalized nonfused multiple GUVs (black) and hemifused GUVs without melittin (blue). The increase of fluorescence intensity at the single GUV level was followed and the error bars represent variations between GUVs during a single experiment. Initial rates; $2.37 \pm 0.48$ and $0.67 \pm 0.16$ a.u for hemifused and non-fused GUVs, respectively.

Given the above observations, we exploited the structurally integrated GUVs for the spatial positioning of enzyme reactions within colonies of the hemifused protocells. For a proof of concept, we implemented a simple and well-established signal transduction pathway into an inter-vesicular enzyme GOx/HRP cascade reaction (Fig. 3a). ${ }^{12,30,31}$ All the GUVs showed similar
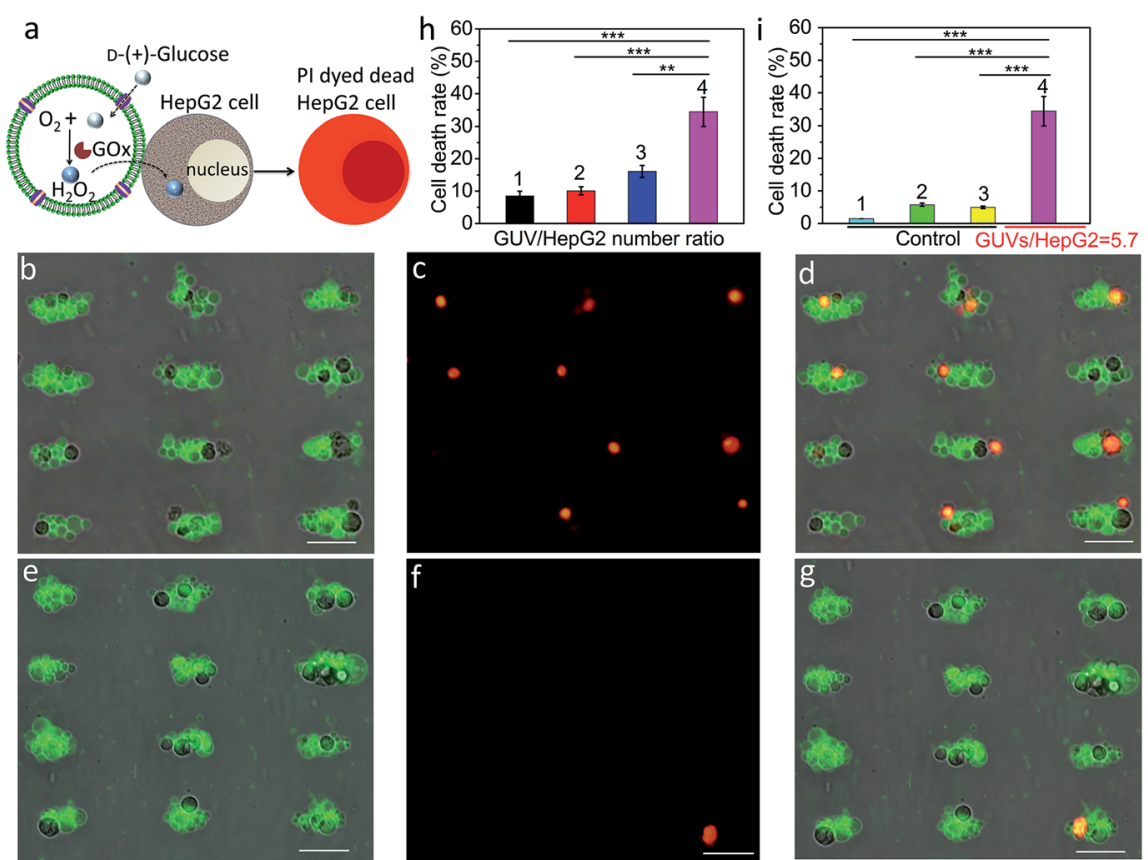

Fig. 4 (a) Schematic showing $\mathrm{H}_{2} \mathrm{O}_{2}$-induced killing of HepG2 cells by co-trapped melittin-functionalized GOx-containing GUVs. (b) Superposition of optical and green fluorescence microscopy images of co-trapped clusters of melittin/GOx-GUVs and HepG2 cells (dark objects) recorded $4 \mathrm{~h}$ after addition of glucose (GUV/HepG2 number ratio, 5.7). (c) Red fluorescence microscopy image of (b) after staining with PI to determine the number of dead cancer cells. (d) Superposition of (b) and (c). (e) Superposition of optical and green fluorescence microscopy images of co-trapped clusters of melittin/GUVs and HepG2 cells (dark objects) without GOx encapsulated in the GUVs recorded $4 \mathrm{~h}$ after addition of glucose (GUV/HepG2 number ratio, 5.7). (f) Red fluorescence microscopy image of (e) after staining with PI to determine the number of dead cancer cells. (g) Superposition of (e) and (f). Scale bars in (b-g) are $50 \mu \mathrm{m}$. (h) Corresponding histogram of percentage of dead HepG2 cells after $4 \mathrm{~h}$ for GUV/HepG2 number ratios of 0.5 (1), 1.4 (2), 2.9 (3) and 5.7 (4). Approximately 56000 cells were used in each experiment; $(n=3$, $* * p<0.01, * * * p<0.001$ ). (i) Control experiments undertaken at a GUV/HepG2 number ratio of 5.7; histograms showing low percentage ( $<6 \%$ ) of dead cells after $4 \mathrm{~h}$ for HepG2 cells alone (1), HepG2/melittin-GUVs (no GOx) (2) and HepG2/GOx-GUVs (no melittin) (3) compared with HepG2/ melittin/GOx-GUVs $(4) ;(n=3, * * * p<0.001)$. Three independent replicates were conducted in each experimental group and control group. Error bars represent the standard deviation. 
enzyme concentration, and the encapsulation efficiency was independent on the size of GUVs (Fig. S15 $\dagger$ ). The cascade was triggered by the spontaneous membrane insertion of a toroidal pore-forming cationic peptide (melittin) ${ }^{32}$ and concomitant uptake of glucose into the GUVs. A red fluorescence output was detected specifically in the HRP-loaded but not in the cotrapped GOx-containing hemifused GUVs within a few minutes in the presence of melittin, Amplex red and glucose (Fig. 3b-d, and S16 $\dagger$ ), consistent with facile penetration of glucose through the peptide channels and inter-vesicular transfer of $\mathrm{H}_{2} \mathrm{O}_{2}$. No fluorescent output was observed in hemifused colonies of co-trapped GUVs that did not include melittin (Fig. S17†) or both types of enzymes (Fig. S18 $\dagger$ ), indicating that uptake of glucose through the peptide channels implemented a localized pathway for signal transduction specifically between the different protocells. Similar observations were made for resorufin production in non-fused aggregates of co-trapped GUVs comprising mixed populations of melittin-loaded enzyme-containing vesicles (Fig. S19†). However, the rate of resorufin production measured at a similar enzyme loading, substrate concentration and GUV number ratio per node was considerably lower in the non-fused GUVs compared with the hemifused arrangement (Fig. 3e). We attributed this to increased diffusional constraints associated with the transfer of $\mathrm{H}_{2} \mathrm{O}_{2}$ across two separated bilayers for colonies of non-fused GUVs compared with the single bilayer connections present in the hemifused protocells.

We employed a similar signal-induced process for the localization and killing of cancer cells by co-trapping clusters of melittin-functionalized GOx-containing GUVs and HepG2 cells at the acoustic pressure nodes (Fig. 4a). As shown by optical and fluorescence microscopy, co-localized clusters of NBD-PE labeled melittin/GOx GUVs (green fluorescence) and propidium iodide (PI)-stained HepG2 cells displayed significant numbers of dead cells at the acoustic nodes (Fig. 4b-d) compared to the control experiments in which clusters of GUVs and HepG2 cells were co-cultured in the absence of GOx in the GUVs (Fig. 4e-g), indicating that toxic levels of hydrogen peroxide were transferred into the adjacent cells. Increasing the number of GUVs per node increased the killing efficiency, consistent with the increased flux of hydrogen peroxide under these conditions (Fig. S20†). As a consequence, over $35 \%$ of the trapped HepG2 cells were killed in a period of $4 \mathrm{~h}$ at a GUV/ HepG2 number ratio of 5.7 (Fig. $4 \mathrm{~h}$ and S21†). Control experiments undertaken in the absence of encapsulated GOx or melittin, or without GUVs showed minimal levels of cell death (Fig. $4 \mathrm{i}$ and $\mathrm{S} 22 \dagger$ ).

Finally, we explored the possibility of producing integrated protocell/living cell microarrays by co-locating clusters of GUVs and $E$. coli cells at the acoustic pressure nodes and exploiting the mutual organization for protocell-mediated induction of bacterial gene expression (Fig. 5a). ${ }^{33-36}$ To achieve this, we encapsulated IPTG in the GUVs prior to acoustic trapping with a population of $E$. coli BL21(DE3) pLysS cells transformed with a plasmid encoded for green fluorescent protein (GFP) expression under a T7 promoter/lac operator. Melittin was added to initiate membrane-mediated transfer of IPTG from the GUVs to neighbouring $E$. coli, which in turn resulted in GFP expression in the bacterial cells (Fig. 5b-d and S23-25†). In contrast, control experiments showed that there was no GFP expression in $E$. coli in the absence of melittin suggesting that there was no passive leakage of IPTG from the GUVs (Fig. 5f-h). Moreover, a IPTG OE.coli E.coli with GFP IIMelittin pore

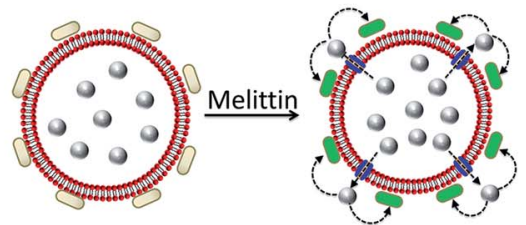

e

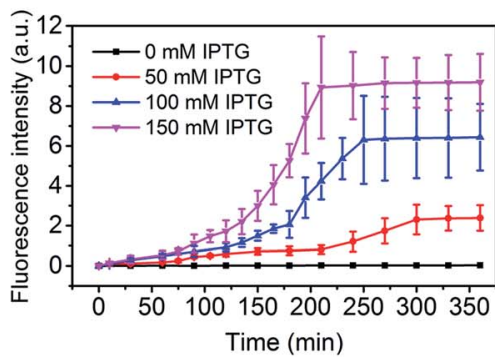

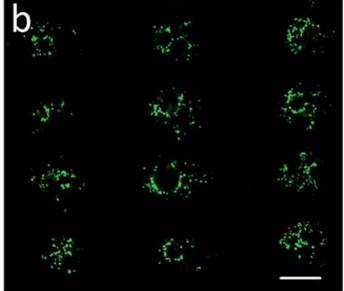
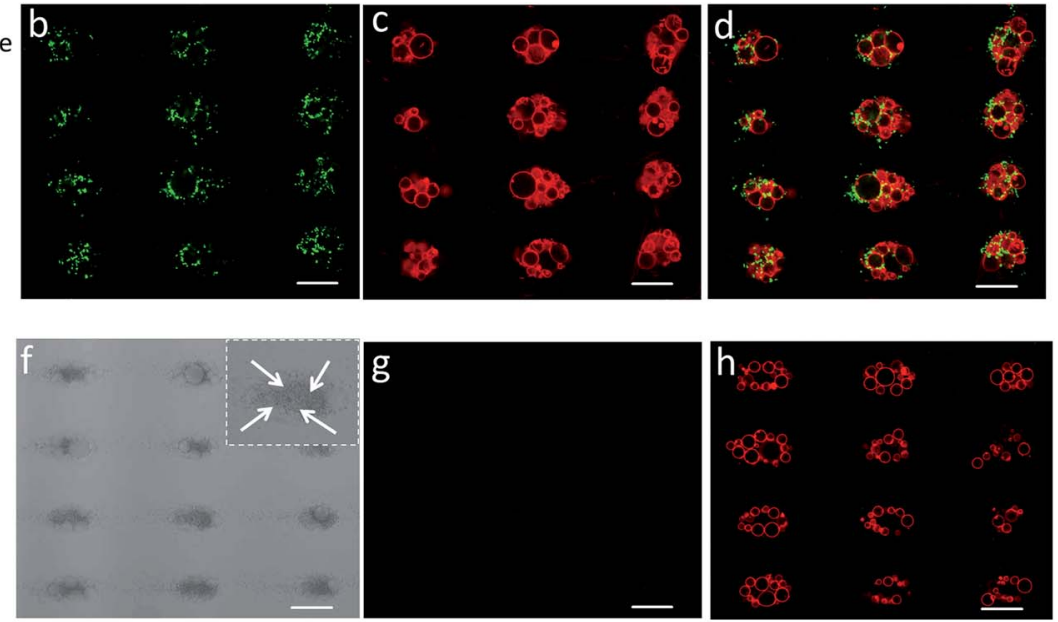

Fig. 5 (a) Schematic showing IPTG-induced GFP expression in E. coli by co-trapped melittin-functionalized IPTG-containing GUVs. (b-d) Representative fluorescence microscopy images of GFP expression in E. coli cells (green fluorescence) (b), co-trapped GUVs (red fluorescence, $0.5 \%$ TR-DHPE) (c) and green/red superimposition (d) of co-trapped clusters of IPTG (150 mM)-containing GUVs and E. coli recorded 210 min after addition of melittin. (e) Time-dependent changes in mean fluorescence intensity for IPTG-induced GFP expression in E. coli by co-trapped melittin-functionalized GUVs containing IPTG with different concentrations. ( $f-h)$ Representative bright field image (f) and fluorescence microscopy images recorded at $463 \mathrm{~nm}$ excitation (green fluorescence) (g) and $535 \mathrm{~nm}$ (red fluorescence, 0.5\% TR-DHPE labelled GUVs) (h) of co-localized IPTG containing GUVs and E. coli recorded $6 \mathrm{~h}$ without melittin. All scale bars are $50 \mu \mathrm{m}$. The darker dots observed at the nodes (white arrows in the inset in (f)) are E. coli cells. Image g indicates no GFP expression in $E$. coli in the absence melittin. Scale bars are $50 \mu m$. 
accumulation of bacteria at the GUV membrane in the acoustic field did not lead to pore formation on the lipid membrane. An IPTG concentration-dependent response was observed in the bacterial cells with maximum levels of GFP expression being observed typically over a period of $200 \mathrm{~min}$ for entrapped inducer concentrations of $150 \mathrm{mM}$ (Fig. 5e, S26, and S27†).

\section{Conclusion}

In conclusion, 1D and 2D micro-arrays of GUVs with controllable spatial geometries, lattice spacings and number of vesicles per pressure node are fabricated by acoustic trapping. The methodology is straightforward, adaptable in terms of microengineering, and readily amenable to in situ chemical manipulations possibly including non-equilibrium spatiotemporal sensing. ${ }^{30}$ Spatially arranged clusters of GUVs can be used to produce hemifused colonies of co-trapped vesicles capable of localized enzyme-directed chemical communication via peptide-mediated inter-vesicular diffusion. Similarly, clusters of HepG2 cells and GUVs are employed as platforms for the signalinduced enzyme-mediated killing of co-trapped cancer cells. Additionally, micro-arrays of IPTG-containing GUVs and E. coli cells can be employed as transmitter/receiver platforms for the local induction of GFP expression, suggesting a step towards high throughput chemical signalling between artificial cells and living cells. Our results are relevant for research in bottom-up synthetic biology, ${ }^{19,20,37}$ including the assembly of synthetic prototissues, ${ }^{38-41}$ and design of vesicle-mediated chemical communication pathways in protocell/living cell consortia. ${ }^{33,36,42,43}$ A key advantage of the described methodology is that it provides a quick access to various arrays of GUVs or GUVs/cells, which in principle could be adapted as multifunctional high throughput platforms for chemical and biochemical screening, enzymatic assays and clinical diagnostics. For instance, chemical signal transfer within vesicle colonies could be explored in heterogeneous multi-vesicle arrays to implement cooperative inter-cellular communications. Moreover, arrays of hybrid vesicles and living cells provide opportunities for the rapid screening of diverse cell-lipid vesicle interactions.

\section{Experimental}

\section{Materials}

1,2-Dioleoyl-sn-glycero-3-phosphocholine (DOPC) was obtained from Avanti Polar Lipids; Texas Red-labeled 1,2-dihexadecanoyl$s n$-glycero-3-phosphoethanolamine triethylammonium salt (TRDHPE) and $N$-(7-nitrobenz-2-oxa-1,3-diazol-4-yl)-1,2-dihexadecanoyl-sn-glycero-3-phosphoethanolamine triethylammonium salt (NBD-PE) were supplied by Thermal Fisher Scientific; D-(+)-glucose $(\geq 99.5 \%)$, chloroform, melittin ( $\geq 85 \%$ HPLC, Sigma), Amplex red ( $\geq 98.0 \%$ HPLC), glucose oxidase (GOx) from Aspergillus niger (type VII, $\geq 100000$ units per g solid), horseradish peroxidase (HRP) ( $\geq 250$ units per $\mathrm{g}$ solid), bovine serum albumin (BSA), rhodamine- $\mathrm{B}$, rhodamine-isothiocyanate (RITC), fluorescein-isothiocyanate (FITC) and isopropyl $\beta$-Dthiogalactoside (IPTG) were purchased from Sigma Aldrich.
Glass slides coated with indium tin oxide (ITO, sheet resistance $\approx 8$ to $12 \Omega$ per square, thickness $\approx 160 \mathrm{~nm}$ ) were produced by Hangzhou Yuhong Technology. Saline solution and sucrose were supplied by Xilong Chemicals. Millipore MilliQ water with a resistivity of $18.0 \mathrm{M} \Omega \mathrm{cm}$ was used throughout.

\section{Preparation of sucrose-containing GUVs}

GUVs were prepared using an electroformation method. In general, a chloroform solution of the lipids (DOPC, $8 \mu \mathrm{L}, 5 \mathrm{mg}$ $\mathrm{mL}^{-1}$ ) labelled with the fluorescent lipids TR-DHPE $0.5 \%$ (red fluorescence) or NBD-PE 5\% (green fluorescence) was deposited on two indium tin oxide (ITO) coated glass slides, and then spread gently on the conductive side of ITO. The residual chloroform was removed under a nitrogen flow. The two ITOcoated glasses were separated by a polytetrafluoroethylene (PTFE) spacer to form an electroformation chamber, which was filled with sucrose solution (0-400 mM). An AC electric field $(10 \mathrm{~Hz}, 5 \mathrm{~V})$ was then applied by a signal generator for $2 \mathrm{~h}$ to produce a stock dispersion of the sucrose-containing GUVs. The number of GUVs per unit volume (vesicle density) was obtained as follows. $10 \mu \mathrm{L}$ of a sucrose-containing GUVs stock solution was added to $120 \mu \mathrm{L}$ of isotonic glucose solution, and placed into the observation chamber of a fluorescence microscope and the GUVs allowed to sediment onto the substrate (10 $\mathrm{min})$. Fluorescence microscopy images were then arbitrarily recorded and the GUV density $(\rho)$ was calculated from $\rho=\sigma / V \times A_{\mathrm{S}} / A_{\mathrm{F}}$, where $\rho$ represents the vesicle density, $\sigma$ the average number of the GUVs in the field of view, $V$ is the volume $(10 \mu \mathrm{L})$ of added GUVs to the chamber, $A_{\mathrm{S}}$ is the total area of the observation chamber, and $A_{\mathrm{F}}$ is the area of the field of view. Typically, the value of $\rho$ was $2152 \pm 110$ per $\mu \mathrm{L}$, which was consistent with flow cytometry results (BD Accuri C6 flow cytometer).

\section{Enzyme encapsulation in GUVs}

$0.05 \mathrm{mg} \mathrm{mL}^{-1}$ RITC-HRP or HRP $\left(0.1 \mathrm{U} \mathrm{mL}^{-1}\right)$ were dissolved in a $400 \mathrm{mM}$ aqueous sucrose solution and encapsulated in DOPC GUVs by electroformation. HRP-containing GUVs were washed three times with isotonic glucose to remove external HRP, and monitored by UV/vis spectroscopy (280 nm) and fluorescence microscopy. Alternatively, $0.05 \mathrm{mg} \mathrm{mL}^{-1}$ FITC-GOx or GOx (1 U $\mathrm{mL}^{-1}$ ) were dissolved in a $400 \mathrm{mM}$ aqueous sucrose solution and encapsulated in DOPC GUVs by electroformation in the presence of dextran (65 $\mathrm{mg} \mathrm{mL}^{-1}$ ). GOx in the external phase was removed using a Sephadex G150 column loaded with sucrose, or by centrifugation and washing with isotonic sucrose solution. Removal of GOx from the external phase was monitored by UV/vis spectroscopy (280 $\mathrm{nm}$ ) or by using an Amplex red assay of the supernatant.

\section{Operation of the acoustic trapping device}

The GUV micro-array was prepared in a custom-built acoustic trapping device with a square arrangement of four piezoelectric transducers (PZT, Noliac, NCE 51, L15 $\times$ W2 mm) or a hexagonal arrangement of six PZTs. The orthogonal transducer pairs were wired in series, driven with a sinusoidal voltage $(10 \mathrm{~V})$ by a signal generator (Agilent 33220a-001), and connected to an 
oscilloscope (Agilent DSOX2014A). A bovine serum albumin (BSA)-modified glass coverslip was attached with adhesive to the bottom of the device. Transducer pairs were operated at their resonance frequency $(5.06,6.71$ or $9.13 \mathrm{MHz}$ for 1D patterns; 6.69/6.71 MHz for the square pattern; 5.06/9.13 $\mathrm{MHz}$ for the rectangular pattern; 6.70/6.71/6.72 $\mathrm{MHz}$ for the hexagonal pattern) with corresponding wavelengths of 162,146 or $110 \mu \mathrm{m}$, $221 / 220 \mu \mathrm{m}, 292 / 162 \mu \mathrm{m}$ and 221.96/220.63/220.30 $\mu \mathrm{m}$, respectively.

\section{Hemifusion of acoustically trapped GUVs}

Hemifusion of co-trapped GUVs prepared with different fluorescent labels was induced by addition of aqueous $\mathrm{CaCl}_{2}(10 \mu \mathrm{L}$, $133 \mathrm{mM}$ ) to the acoustic trapping chamber in the presence of an applied acoustic field. Exchange of lipids between the adjacent vesicles was observed after 20 minutes.

\section{Dynamic modulation of GUV array}

The GUV array was prepared by adding $30 \mu \mathrm{L}$ of sucrose containing GUVs (400 mM) to isotonic glucose solution ( $1 \mathrm{~mL}, 400$ $\mathrm{mM}$ ). To control dynamic modulation of the GUV array, the two orthogonal pairs of transducers were switched on/off every $30 \mathrm{~s}$. For the dynamic modulation of a hemifused/non-hemifused GUV array, a 2D array of TR-DHPE labelled GUVs (red) was firstly prepared by adding $30 \mu \mathrm{L}$ of sucrose containing GUVs $(400 \mathrm{mM})$ to isotonic glucose solution $(1 \mathrm{~mL}, 400 \mathrm{mM})$ in the presence of a 2D acoustic pressure field $(5.06 / 9.13 \mathrm{MHz}, 10 \mathrm{~V})$, and then was hemifused by the additional of $\mathrm{CaCl}_{2}(10 \mu \mathrm{L}, 133$ $\mathrm{mM}$ ) in the same acoustic pressure field. Then, the supernatant in the acoustic trapping chamber was carefully replaced by glucose solution $(400 \mathrm{mM})$ to remove the $\mathrm{Ca}^{2+}$. The hemifused/ non-hemifused hybrid GUV array was obtained by the addition of $20 \mu \mathrm{L}$ of sucrose containing NBD-PE labelled GUV (green) to the acoustic trapping chamber. To control dynamic modulation of the GUV array, the two orthogonal pairs of transducers were switched on/off about every $60 \mathrm{~s}$.

\section{Enzyme cascade reactions in acoustically trapped GUV micro- arrays}

Sucrose-containing GOx-(1 $\left.\mathrm{U} \mathrm{mL}^{-1}\right)$ GUVs were co-trapped with sucrose-containing HRP- $\left(0.1 \mathrm{U} \mathrm{mL}^{-1}\right)$-loaded GUVs in the presence of isotonic glucose $(400 \mathrm{mM})$ and Amplex red $(10 \mu \mathrm{L}$, $50 \mu \mathrm{M})$. A signal transduction pathway between the different GUVs before or after $\mathrm{Ca}^{2+}$-induced hemifusion was initiated by addition of melittin (final concentration, $4 \mu \mathrm{g} \mathrm{mL}{ }^{-1}$ ), which inserted into the vesicle bilayers and promoted the glucose influx required for the GOx/HRP enzyme cascade. Location of the individual GOx- and HRP-containing GUVs in the colocalized clusters was determined using a mixture of NBD-PElabelled HRP-containing GUVs (green fluorescence) and unlabeled GOx-loaded GUVs, and identifying the spatial positions of the different GUVs by a combination of optical and fluorescence microscopies. Formation of resorufin specifically in the HRPcontaining GUVs was monitored by fluorescence microscopy $(560 \mathrm{~nm})$.

\section{Signal transduction in co-trapped GUV/HepG2 cell assemblies}

A slightly modified electroformation method was used to prepare GOx-containing GUVs that were functionalized with melittin prior to acoustic trapping with living HepG2 cells. This was done to prevent deleterious effects on the cell membrane accompanying addition of aqueous melittin to the acoustically trapped GUV/HepG2 assemblies. In addition, dextran was encapsulated along with sucrose and GOx to extend the trapping lifetime of the GUVs, which was otherwise curtailed by efflux of sucrose through the melittin pores in the lipid membrane. Typically, a chloroform solution of DOPC $(8 \mu \mathrm{L}$, $\left.5 \mathrm{mg} \mathrm{mL}{ }^{-1}\right)$, NBD-PE $(5 \%)$ and melittin $\left(5 \mu \mathrm{g} \mathrm{mL}{ }^{-1}\right)$ was deposited on ITO coated glass slides, and a pre-mixed solution of GOx (50 $\left.\mathrm{U} \mathrm{mL}^{-1}\right)$, dextran $\left(0.065 \mathrm{~g} \mathrm{~mL}^{-1}\right)$ and sucrose (300 $\mathrm{mM}$ ) added, followed by electroformation. The obtained GOxcontaining GUV suspension was diluted 3 times in isotonic sucrose $(300 \mathrm{mM})$, and GOx in the supernatant removed by six cycles of centrifugation $(7000 \mathrm{rpm}, 10 \mathrm{~min})$ and washing. The number density of the GOx-containing GUVs was $(5.3 \pm 0.3) \times$ $10^{3} / \mu \mathrm{L}$. To prepare micro-arrays consisting of co-localized clusters of multiple GOx-containing GUVs and living HepG2 cells, different numbers of GUVs ( $c a .2 .6 \times 10^{4}, 7.8 \times 10^{4}, 1.5 \times$ $10^{5}$ and $2.3 \times 10^{5}$ ) were added to a stock suspension of HepG2 cells (ca. $5.6 \times 10^{4}$ cells; $1 \mathrm{~mL}$ cell culture medium) and the mixed suspension placed in the acoustic chamber with two transducer pairs $(5.06 / 9.13 \mathrm{MHz})$. After $20 \mathrm{~min}, 100 \mu \mathrm{L}$ of $300 \mathrm{mM}$ glucose was added into the acoustic chamber to initiate the generation of $\mathrm{H}_{2} \mathrm{O}_{2}$ in the GOx-containing GUVs. The microarrays were incubated for $4 \mathrm{~h}$, and propidium iodine (PI) in PBS buffer solution $\left(20 \mu \mathrm{L}, 20 \mu \mathrm{g} \mathrm{mL}{ }^{-1}\right)$ was then added to the acoustic chamber to stain the dead cells over a period of $20 \mathrm{~min}$. The numbers of dead cells produced after $4 \mathrm{~h}$ were determined from fluorescence microscopy images (excitation wavelength, $536 \mathrm{~nm})$.

\section{Optical and fluorescence microscopy}

Optical microscopy experiments were carried out on a Leica DMI 3000B optical microscope. Fluorescence imaging was performed using a Leica DFC 310FX set up, and dye molecules were excited by using specific filters with the following excitation $\left(\lambda_{\mathrm{ex}}\right)$ and emission wavelength cut offs $\left(\lambda_{\mathrm{em}}\right)$; NBD-PE, $\lambda_{\mathrm{ex}}=$ $463 \mathrm{~nm}, \lambda_{\mathrm{em}}=536 \mathrm{~nm}$; FITC, $\lambda_{\mathrm{ex}}=492 \mathrm{~nm}$ and $\lambda_{\mathrm{em}}=518 \mathrm{~nm}$; TR-DHPE, $\lambda_{\mathrm{ex}}=582 \mathrm{~nm}$ and $\lambda_{\mathrm{em}}=601 \mathrm{~nm}$; rhodamine- $\mathrm{B}, \lambda_{\mathrm{ex}}=$ $528 \mathrm{~nm}$ and $\lambda_{\mathrm{em}}=551 \mathrm{~nm}$; RITC, $\lambda_{\mathrm{ex}}=570 \mathrm{~nm}$ and $\lambda_{\mathrm{em}}=$ $590 \mathrm{~nm}$; resorufin, $\lambda_{\mathrm{ex}}=560 \mathrm{~nm}$ and $\lambda_{\mathrm{em}}=572 \mathrm{~nm}$; propidium iodide (PI), $\lambda_{\mathrm{ex}}=536 \mathrm{~nm}, \lambda_{\mathrm{em}}=$ emission $617 \mathrm{~nm}$. All glass slides used for the imaging were coated with BSA.

\section{HepG2 cell cultures}

HepG2 cells were cultured in medium (Dulbecco's Modified Eagle's Medium; $4.5 \mathrm{~g} \mathrm{~L}^{-1}$ glucose, sodium pyruvate and sodium bicarbonate supplemented with $10 \%$ fetal bovine serum, $2 \mathrm{mM}$ L-glutamine, $50 \mu \mathrm{g} \mathrm{mL} \mathrm{m}^{-1}$ streptomycin and $50 \mu \mathrm{g}$ $\mathrm{mL}^{-1}$ penicillin (all from Sigma-Aldrich)) at $37{ }^{\circ} \mathrm{C}$ and $5 \% \mathrm{CO}_{2}$ atmosphere. The pre-cultured HepG2 cells were digested by 
trypsin, which was removed by centrifugation at $1000 \mathrm{rpm}$ for $3 \mathrm{~min}$, and the concentrated cells were re-dispersed in the medium $(5 \mathrm{~mL})$. The density of HepG2 cells was $3 \times 10^{6} \pm 4 \times$ $10^{5} / \mathrm{mL}$. The HepG2 cells were kept at low temperature by placing tubes of the cell suspensions in ice before trapping.

\section{Preparation of $E$. coli}

E. coli BL21(DE3) pLysS transformed with a plasmid-encoding GFP behind a T7 promoter and a lac operator sequence were incubated in Luria-Bertani (LB) liquid medium (including $10 \mathrm{~g} \mathrm{~L}^{-1}$ tryptone, $5 \mathrm{~g} \mathrm{~L}^{-1}$ yeast extract, and $10 \mathrm{~g} \mathrm{~L}^{-1} \mathrm{NaCl}$ ) for $16 \mathrm{~h}$ at $37^{\circ} \mathrm{C}$.

\section{IPTG-induced gene expression of GFP in co-trapped GUV/E. coli arrays}

IPTG containing GUVs were electroformed $(3.5 \mathrm{~V}, 10 \mathrm{~Hz})$ in different mixtures of IPTG/sucrose mixtures $(50 \mathrm{mM} / 250 \mathrm{mM}$, $100 \mathrm{mM} / 200 \mathrm{mM}, 150 \mathrm{mM} / 150 \mathrm{mM}$ ) for $2 \mathrm{~h}$. The obtained IPTGcontaining GUV suspension was diluted 3 times in isotonic glucose $(300 \mathrm{mM})$ solution. IPTG in the supernatant was removed by six cycles of centrifugation (4000 rpm, $10 \mathrm{~min}$ ) and washing by $300 \mathrm{mM}$ glucose. $E$. coli cells in medium $(3 \mathrm{~mL})$ were concentrated by centrifugation (5000 rpm, $15 \mathrm{~min})$ and redispersed in $300 \mathrm{mM}$ glucose $(400 \mu \mathrm{L})$. In all the GUV/E. coli arrays, $20 \mu \mathrm{L}$ of above-mentioned GUVs glucose solution and 20 $\mu \mathrm{L}$ of above-mentioned $E$. coli glucose solution were added in $1 \mathrm{~mL}$ glucose solution ( $300 \mathrm{mM}$ ) to the acoustic chamber. Signalling between the co-trapped GUVs and E. coli was induced by addition of melittin (final concentration, $4 \mu \mathrm{g} \mathrm{mL} \mathrm{m}^{-1}$ ).

\section{Simulation of the acoustic pressure in the device}

The acoustic pressure field in the device was simulated according to a previous report. ${ }^{44}$ In this simulation, the following assumptions were made: (i) the reflections of the acoustic waves are ignored; (ii) the initial transient period was not considered for the simulation after each opposed transducer pair is excited with a sinusoidal voltage; (iii) the standing wave was treated as the sum of two counter-propagating plane waves and any interference between these two fields averages to zero when each of the transducer pairs were operated at different frequencies. The total acoustic pressure in the devices is then given by the sum of the two standing waves created by the orthogonal pairs:

$$
p=p_{1}\left(\mathrm{e}^{i k_{1} x}+\mathrm{e}^{-i k_{1} x}\right) \mathrm{e}^{i \omega_{1} t}+p_{2}\left(\mathrm{e}^{i k_{2} y}+\mathrm{e}^{-i k_{2} y}\right) \mathrm{e}^{i \omega_{2} t}
$$

where $k_{1}=\frac{\omega_{1}}{c_{0}}$ and $\omega_{1}$ is the angular frequency in $\operatorname{rad~s}^{-1}$ and $c_{0}$ is the speed of sound in the host fluid. The numerical subscript denotes the orthogonal pair under consideration, and is required as the transducer pairs were operated at different frequencies. The Cartesian axes are defined by $x$ and $y$. Transducer pair- 1 creates a standing wave in $x$ whereas transducer pair-2 creates a standing wave in $y$. Gor'kov described the forces as resulting from a potential field, $U$. Using this approach the acoustic radiation force, $\vec{F}$, can be found from

$$
\vec{F}=-\nabla U,
$$

$$
\begin{aligned}
& U=\frac{4 \pi}{3} a^{3}\left[f_{1} \frac{1}{2 \rho_{\mathrm{o}} c_{0}^{2}}\left\langle|p|^{2}\right\rangle-f_{2} \frac{3}{4} \rho_{\mathrm{o}}\left\langle|\vec{v}|^{2}\right\rangle\right] \\
& f_{1}=1-\frac{\rho_{\mathrm{o}} c_{0}^{2}}{\rho_{\mathrm{p}} c_{\mathrm{p}}^{2}} \text { and } f_{2}=\frac{2\left(\rho_{\mathrm{p}} / \rho_{\mathrm{o}}-1\right)}{2 \rho_{\mathrm{p}} / \rho_{\mathrm{o}}+1}
\end{aligned}
$$

where $\left\langle|p|^{2}\right\rangle$ and $\left\langle|\vec{v}|^{2}\right\rangle$ are the mean squared pressure and particle velocity respectively at the object, $a$ is the radius of the spherical object, $\rho$ is the density and the subscripts denote the particle, 'p', or host, 'o' properties. Note also, for a harmonic sound field, $\vec{v}=\frac{1}{i \omega \rho_{\mathrm{o}}} \nabla p$.

\section{Conflicts of interest}

There are no conflicts to declare.

\section{Acknowledgements}

This work was supported by the National Natural Science Foundation of China (Grant No. 21773050 and 21929401) and the Natural Science Foundation of Heilongjiang Province for Distinguished Young Scholars (JC2018003). L. T. and S. M. thank BrisSynBio (a BBSRC/EPSRC Synthetic Biology Research Centre; BB/L01386X/1) at the University of Bristol and Engineering and Physical Sciences Research Council (EPSRC, UK; EP/L002957/1) for financial support.

\section{Notes and references}

1 P. Walde, K. Cosentino, H. Engel and P. Stano, ChemBioChem, 2010, 11, 848-865.

2 L. Lu, W. J. Doak, J. W. Schertzer and P. R. Chiarot, Soft Matter, 2016, 12, 7521-7528.

3 Y. S. Ryu, D. Yoo, N. J. Wittenberg, L. R. Jordan, S. D. Lee, A. N. Parikh and S. H. Oh, J. Am. Chem. Soc., 2015, 137, 8692-8695.

4 K. Karamdad, J. W. Hindley, G. Bolognesi, M. S. Friddin, R. V. Law, N. J. Brooks, O. Ces and Y. Elani, Chem. Sci., 2018, 9, 4851-4858.

5 T. Zhu and J. W. Szostak, J. Am. Chem. Soc., 2009, 131, 57055713.

6 M. Andes-Koback and C. D. Keating, J. Am. Chem. Soc., 2011, 133, 9545-9555.

7 T. Robinson, P. E. Verboket, K. Eyer and P. S. Dittrich, $L a b$ Chip, 2014, 14, 2852-2859.

8 T. Tanaka and M. Yamazaki, Langmuir, 2004, 20, 5160-5164.

9 N. Mora, A. Bahreman, H. Valkenier, H. Li, T. H. Sharp, D. N. Sheppard, A. P. Davis and A. Kros, Chem. Sci., 2016, 7, 1768-1772.

10 M. Chabanon, J. C. S. Ho, B. Liedberg, A. N. Parikh and P. Rangamani, Biophys. J., 2017, 112, 1682-1691.

11 H. Pick, A. C. Alves and H. Vogel, Chem. Rev., 2018, 118, 8598-8654.

12 Y. Elani, R. V. Law and O. Ces, Nat. Commun., 2014, 5, 5305. 
13 N. N. Deng, M. A. Vibhute, L. Zheng, H. Zhao, M. Yelleswarapu and W. T. S. Huck, J. Am. Chem. Soc., 2018, 140, 7399-7402.

14 R. K. Kumar, X. Yu, A. J. Patil, M. Li and S. Mann, Angew. Chem., Int. Ed., 2011, 50, 9343-9347.

15 W. Zong, S. H. Ma, X. N. Zhang, X. J. Wang, Q. C. Li and X. J. Han, J. Am. Chem. Soc., 2017, 139, 9955-9960.

16 M. Weiss, J. P. Frohnmayer, L. T. Benk, B. Haller, J. Janiesch, T. Heitkamp, M. Börsch, R. B. Lira, R. Dimova, R. Lipowsky, E. Bodenschatz, J. C. Baret, T. Vidakovic-Koch, K. Sundmacher, I. Platzman and J. P. Spatz, Nat. Mater., 2018, 17, 89-96.

17 R. Tomasi, J. Noel, A. Zenati, S. Ristori, F. Rossi, V. Cabuil, F. Kanou and A. Hassan, Chem. Sci., 2014, 5, 1854-1859.

18 A. Bhattacharya, R. J. Brea and N. K. Devaraj, Chem. Sci., 2017, 8, 7912-7922.

19 P. Taylor, C. Xu, P. D. I. Fletcher and V. N. Paunov, Phys. Chem. Chem. Phys., 2003, 5, 4918-4922.

20 Y. Kang, H. S. Wostein and S. Majd, Adv. Mater., 2013, 25, 6834-6838.

21 Y. Kazayama, T. Teshima, T. Osaki, S. Takeuchi and T. Toyota, Anal. Chem., 2016, 88, 1111-1116.

22 N. Yandrapalli and T. Robinson, Lab Chip, 2019, 19, 626-633. 23 X. Ding, S. C. S. Lin, B. Kiraly, H. Yue, S. Li, I. K. Chiang, J. J. Shi, S. J. Benkovic and T. J. Huang, Proc. Natl. Acad. Sci. U. S. A., 2012, 109, 11105-11109.

24 B. W. Drinkwater, Lab Chip, 2016, 16, 2360-2375.

25 L. Tian, N. Martin, P. G. Bassindale, A. J. Patil, M. Li, A. Barnes, B. W. Drinkwater and S. Mann, Nat. Commun., 2016, 7, 13068.

26 D. J. Collins, B. Morahan, J. Garcia-Bustos, C. Doerig, M. Plebanski and A. Neild, Nat. Commun., 2015, 6, 8686.

27 P. Carrara, P. Stano and P. L. Luisi, ChemBioChem, 2012, 13, 1497-1502.

28 C. Zhu, E. M. Taipaleenmäki, Y. Zhang, X. Han and B. Städler, Biomater. Sci., 2018, 6, 793-802.

29 H. M. Bi, B. Yang, L. Wang, W. Cao and X. J. Han, J. Mater. Chem. A, 2013, 1, 7125-7130.
30 L. Tian, M. Li, J. B. Liu, A. J. Patil, B. W. Drinkwater and S. Mann, ACS Cent. Sci., 2018, 4, 1551-1558.

31 S. Li, X. Wang, W. Mu and X. Han, Anal. Chem., 2019, 9, 6859-6864.

32 M. Mally, J. Majhenc, S. Svetina and B. Zeks, Biochim. Biophys. Acta, Biomembr., 2007, 1768, 1179-1189.

33 R. Lentini, S. P. Santero, F. Chizzolini, D. Cecchi, J. Fontana, M. Marchioretto, C. Bianco, J. L. Terrell, A. C. Spencer, L. Martini, M. Forlin, M. Assfalg, M. D. Serra, W. E. Bentley and S. S. Mansy, Nat. Commun., 2014, 5, 4012.

34 G. Rampioni, F. D'Angelo, M. Messina, A. Zennaro, Y. Kuruma, D. Tofani, L. Leoni and P. Stano, Chem. Commun., 2018, 54, 2090-2093.

35 R. Lentini, N. Y. Martín, M. Forlin, L. Belmonte, J. Fontana, M. Cornella, L. Martini, S. Tamburini, W. E. Bentley, O. Jousson and S. S. Mansy, ACS Cent. Sci., 2017, 3, 117-123.

36 T. Trantidou, L. Dekker, K. Polizzi, O. Ces and Y. Elani, Interface Focus, 2018, 8, 20180024.

37 A. Joesaar, S. Yang, B. Bögels, A. Linden, P. Pieters, B. V. V. S. P. Kumar, N. Dalchau, A. Phillips, S. Mann and T. F. A. de Greef, Nat. Nanotechnol., 2019, 14, 369-378.

38 P. Gobbo, A. J. Patil, M. Li and S. Mann, Nat. Mater., 2018, 17, 1145-1153.

39 M. J. Booth, V. R. Schild, A. D. Graham, S. N. Olof and H. Bayley, Sci. Adv., 2016, 2, e1600056.

40 G. Bolognesi, M. S. Friddin, A. Salehi-Reyhani, N. E. Barlow, N. J. Brooks, O. Ces and Y. Elani, Nat. Commun., 2018, 9, 1882.

41 Y. Elani, A. Gee, R. V. Law and O. Ces, Chem. Sci., 2013, 4, 3332-3338.

42 P. M. Gardner, K. Winzer and B. G. Davis, Nat. Chem., 2009, 1, 377-383.

43 A. X. Lu, H. Oh, J. L. Terrell, W. E. Bentley and S. R. Raghavan, Chem. Sci., 2017, 8, 6893-6903.

44 C. R. P. Courtney, C. K. Ong, B. W. Drinkwater, A. L. Bernassau, P. D. Wilcox and D. R. S. Cumming, Proc. R. Soc. Edinburgh, Sect. A: Math. Phys. Sci., 2011, 468, 337360. 